\title{
Inserción de la temática Gestión de Riesgo y Cambio Climático con enfoque de género y gobernabilidad, como eje transversal, en la asignatura metodología de la investigación
}

Franklin Solís Zúniga ${ }^{1}$

1 UNAN-Managua, FAREM-Estelí. Correo electrónico: franksolis23@yahoo.com

\section{RESUMEN}

La experiencia de investigación que aquí se presenta, está orientada al tema de la inserción de ejes transversales y atiende a una habilidad formativa cada vez más necesaria en el proceso de formación profesional de los/as estudiantes. Consiste en la incorporación de la temática gestión integral de riesgo y cambio climático con enfoque de género y gobernabilidad, como eje transversal, en la unidad dos, proceso de la investigación científica de la Asignatura Metodología de la Investigación de la Carrera de Enseñanza de las Ciencias Naturales del turno sabatino, durante los meses de abril a junio del año 2013.

Esta investigación se realizó a partir de un diseño cualitativo desde la perspectiva de la Investigación Acción. Las técnicas aplicadas para la recolección de la información fueron, la observación, grupos de discusión, el diario de campo, los registros fotográficos y la entrevista como evaluación final. La información obtenida con las técnicas aplicadas fue analizada en función de los objetivos formulados y según su naturaleza cualitativa.

Como resultado principal del trabajo realizado se obtuvo la integración del eje transversal de gestión de riesgo y cambio climático con enfoque de género y gobernabilidad, en la mayoría de las temáticas de investigación elegidas por los grupos de estudiantes. Por tanto, se puede decir que se logró comprobar la hipótesis de acción planteada.

Palabras clave: Eje transversal, Gestión de riesgo, cambio climático, género, gobernabilidad, Metodología de la investigación. 


\title{
Inserting the topic risk management and climate change with gender approach and governance as cross shaft in the Methodology research course
}

Franklin Solís Zúniga ${ }^{1}$

1 UNAN-Managua, FAREM-Estelí. E-mail: franksolis23@yahoo.com

\begin{abstract}
Experience research presented here is oriented to the issue of the inclusion of crosscutting and serves increasingly necessary skill training in the process of professional training of the students. It consists in the incorporation of thematic comprehensive risk management and climate change with gender approach and governance as a transversal axis in unit two, "Process of scientific research, Methodology Course of the Career of Teaching Nature Science of the Saturday shift, during the months of April to June 2013.

This research was conducted from a qualitative design from the perspective of action research. The techniques used for data collection were, observation, discussion groups, diary, photographic records and interview as final evaluation. The information obtained with the applied techniques was analyzed in terms of the stated objectives and according to their qualitative caracteristics.

The main result of this research was the integration of the transverse axis of risk management and climate change governance and gender approach. It was obtained in most of the research topics chosen by the student teams. Therefore, it was possible to test the hypothesis posed action.
\end{abstract}

Keywords: Cross shaft, risk management, climate change, gender, governance, methodology research. 


\section{INTRODUCCIÓN}

El cambio climático global es un hecho innegable y de larga manifestación, las emisiones de hoy generan peligros y amenazas futuras y los efectos de hoy son consecuencias de lo que se emitió en el pasado. Nuestro país Nicaragua, debido a su ubicación geográfica y a sus condiciones de vulnerabilidad, ha sufrido en el pasado los efectos devastadores de la variabilidad natural del clima, lo que ha forzado a la sociedad a la búsqueda de respuestas de adaptación para atenuar los impactos originados por esos cambios. Inundaciones, huracanes y tormentas se han convertido en la pesadilla del país, empeoradas por el calentamiento de la tierra. Milán (2009). Bajo estas consideraciones, se hace necesario conocer los antecedentes históricos de los desastres, para retomar lecciones aprendidas que permitan en el presente hacer una buena gestión de los riesgos y reducir los desastres a escala nacional. (Blandón, 2013).

De ahí la necesidad de formar a nuestros futuros profesionales en esta temática, con el único fin de contribuir al cambio de actitudes que permitan una gestión adecuada de los riesgos y hacerle frente a los efectos del cambio climático, ya que la educación juega un papel muy importante, pues a través de la información, la constante actualización, las capacitaciones, las personas estaríamos menos expuestas al riesgo, seríamos menos vulnerables, tendríamos una sociedad resiliente.

Por las razones antes mencionadas, la Facultad Regional Multidisciplinaria, FAREM Estelí, a través del Centro de Formación y Capacitación Permanente para la Gestión Integral de Riesgos y Desastres, está promoviendo la capacitación y formación docente de las Facultades de la UNAN Managua sobre el eje transversal de Gestión Integral de Riesgo y Cambio Climático, para que a su vez éste sea insertado en el currículo universitario.
La experiencia de Investigación-Acción que aquí se presenta se enmarca dentro de la posición de la inserción de ejes transversales en la educación superior, pretendiendo ofrecer una pequeña aportación a esta línea de investigación educativa emergente. Esta propuesta de Investigación-Acción se suma a las existentes, siendo el tema objeto de estudio la Inserción de la temática gestión integral de riesgo y cambio climático con enfoque de género y gobernabilidad, como eje transversal, en la Asignatura Metodología de la Investigación. Es por ello que su propósito no es otro que, experimentar la integración de la temática de gestión de riesgo y cambio climático con enfoque de género y gobernabilidad, como eje transversal en la Asignatura Metodología de la Investigación de la carrera de Enseñanza de las Ciencias Naturales del turno sabatino, durante el primer semestre 2013, haciendo uso de algunas pautas metodológicas para aplicarlo al contexto del aula, específicamente en la unidad dos del programa de la Asignatura: Proceso de la Investigación Científica.

El grupo clase objeto de análisis lo constituyen los 45 estudiantes del tercer año de Licenciatura en Enseñanza de las

Ciencias Naturales del turno sabatino, a quienes se les impartía dicha Asignatura. Cabe destacar, que la elección de este grupo no ha sido arbitraria, todo lo contrario, responde a la necesidad de sensibilizar a este grupo de estudiantes acerca de la problemática de gestión de riesgo y cambio climático que afecta a la región, a nuestro país y al mundo entero, mediante la autonomía que ofrece a la Asignatura Metodología de la Investigación y aprovechando sus conocimientos previos.

Finalmente, conviene aclarar que la parte práctica de la investigación gira en torno a los objetivos de Actuación, para ello inicialmente se partió de la elaboración y ejecución de un Plan de Acción o Propuesta de Actuación previamente establecida, 
del cual se derivan los resultados obtenidos con la experiencia.

\section{METODOLOGÍA}

Esta experiencia corresponde al enfoque de Investigación cualitativa, siendo su carácter el de la Investigación-Acción- Participación, metodología que permite desarrollar un análisis participativo, donde los actores implicados, se convierten en los protagonistas del proceso de construcción del conocimiento de la realidad sobre el objeto de estudio, en la detección de problemas y necesidades y en la elaboración de propuestas y soluciones. En ella participaron 45 estudiantes del tercer año de la Licenciatura en Enseñanza de las Ciencias Naturales.

A su vez, el planteamiento de dicha experiencia, se enmarca en el campo de la Investigación Educativa, en este sentido se aborda como una InvestigaciónAcción en Educación. John Elliot, (1993, citado por Herrera, 2013), señala que la InvestigaciónAcción aplicada a la educación tiene que ver con los problemas prácticos cotidianos experimentados por los docentes, más que con problemas teóricos definidos por investigadores dentro de un área del conocimiento.

El proceso de investigación-Acción se llevó a cabo a través de una espiral de ciclos de investigación y acción constituidos por las siguientes fases: Planificación, actuación, observación y reflexión. Inicialmente se partió de sentir experimentar un problema o necesidad a través de una diagnosis, seguidamente, se elaboró y ejecutó un plan de acción y finalmente se evaluaron los resultados obtenidos.

Los acontecimientos relevantes de las diferentes sesiones se obtuvieron a través de técnicas cualitativas de recolección de datos tales como la observación, grupos de discusión, diario de campo, registros fotográficos y de manera complementaria se aplicó una especie de entrevista abierta como evaluación final. La información obtenida con las diferentes técnicas e instrumentos utilizados, fue transcrita y organizada en tablas de doble entrada y se analizó haciendo uso de uno de los métodos más conocidos para el procesamiento y análisis de los datos cualitativos: el análisis de contenido.

\section{RESULTADOS Y DISCUSIÓN}

\section{Resultados de la etapa diagnóstica}

Para cumplir con etapa diagnóstica se procedió a la elaboración de una guía de entrevista que consta de seis preguntas abiertas, las cuales fueron diseñadas con el propósito de valorar las conocimientos y actitudes de los/as estudiantes de la carrera de Ciencias Naturales sobre la problemática de gestión de riesgo y cambio climático. Estas preguntas fueron desarrolladas a través de un grupo de discusión. Es importante destacar, que la realización de la diagnosis tuvo en cuenta los siguientes aspectos que guiaron el proceso de reflexión:

- ¿Qué concepciones y actitudes tienen los/as estudiantes de la carrera de Ciencias Naturales sobre gestión de riesgo y cambio climático?

- ¿Qué factores de riesgo y/o efectos del cambio climático identifican los/as estudiantes en su comunidad?

- ¿Cuál es la reflexión crítica que hacen los/ as estudiantes ante situaciones de riesgo que se puedan presentar en su comunidad?

- ¿De qué manera la asignatura Metodología de la Investigación puede aportar a la gestión adecuada de riesgos y a enfrentar los efectos del cambio climático?

Cuadro $\mathbf{N}^{\circ} \mathbf{1}$. Análisis cualitativo de las concepciones y actitudes de los/as estudiantes de la carrera de Ciencias Naturales sobre la problemática de gestión de riesgo y cambio climático. 


\begin{tabular}{|c|c|}
\hline ASPECTOS CONSULTADOS & $\begin{array}{l}\text { OPINIONES DE LOS Y LAS } \\
\text { ESTUDIANTES }\end{array}$ \\
\hline $\begin{array}{l}\text { Conocimiento sobre gestión } \\
\text { de riesgo y cambio climático. }\end{array}$ & $\begin{array}{l}\text { Gestión de riesgo: "Acciones } \\
\text { y estrategias planificadas y } \\
\text { organizadas enfocadas en } \\
\text { la prevención y mitigación } \\
\text { de fenómenos naturales y } \\
\text { antrópicos". } \\
\text { Cambio climático: } \\
\text { "Alteraciones en la naturaleza } \\
\text { provocadas por el hombre } \\
\text { como consecuencia del uso } \\
\text { irracional de los recursos } \\
\text { naturales". }\end{array}$ \\
\hline $\begin{array}{l}\text { Acciones que podría realizar } \\
\text { como futuro profesional de la } \\
\text { Enseñanza de las Ciencias } \\
\text { Naturales para contribuir a la } \\
\text { gestión adecuada de riesgos y } \\
\text { hacerle frente a los efectos del } \\
\text { cambio climático en nuestro } \\
\text { país. }\end{array}$ & $\begin{array}{l}\text { "Cambio de actitud personal, } \\
\text { promover el uso de las tres } \\
\text { R vitales (Reciclaje, Reusar, } \\
\text { Reducir)", "Priorizar en los } \\
\text { planes de clase actividades } \\
\text { que permitan incluir el eje de } \\
\text { gestión de riesgo". "Sensibilizar } \\
\text { a la población sobre los } \\
\text { daños que le causamos al } \\
\text { medio, promover campañas } \\
\text { educativas" "Formar brigadas } \\
\text { de seguridad escolar". }\end{array}$ \\
\hline $\begin{array}{l}\text { Contribución a la gestión } \\
\text { adecuada de riesgos y a la } \\
\text { reducción de los efectos del } \\
\text { cambio climático desde la } \\
\text { Asignatura de Metodología de } \\
\text { la Investigación. }\end{array}$ & $\begin{array}{l}\text { "Impulsando trabajos } \\
\text { investigativos que ayuden en } \\
\text { la divulgación y promoción de } \\
\text { prevención de desastres y el } \\
\text { cambio climático". "Insertando } \\
\text { la temática en los diferentes } \\
\text { trabajos de curso, ya que } \\
\text { vivimos en un país vulnerable". } \\
\text { "A través del trabajo que } \\
\text { estamos realizando podemos } \\
\text { contribuir a hacer más pública } \\
\text { la información sobre el } \\
\text { enfoque de gestión de riesgo. } \\
\text { También podemos contribuir } \\
\text { a la elaboración de planes de } \\
\text { seguridad". }\end{array}$ \\
\hline
\end{tabular}

Los resultados del diagnóstico demuestran que los y las estudiantes reflejan un adecuado conocimiento y una actitud positiva ante la problemática de gestión de riesgo y cambio climático. Esta situación puede atribuirse a las características particulares de la carrera que estudian, pues al ser estudiantes de Ciencias Naturales, dominan muchos aspectos de esta temática. Asimismo, son conscientes de diversas acciones que podrían realizar como futuros profesionales de las Ciencias Naturales, desde la Asignatura de Metodología de la Investigación.

\section{Hipótesis de acción}

En base a los resultados del diagnóstico se planteó la siguiente hipótesis de acción:

"Si los/as estudiantes del tercer año de la carrera de Ciencias Naturales, aplican el proceso de Investigación Científica en problemas relacionados a la gestión de riesgo y cambio climático con enfoque de género y gobernabilidad, desde la Asignatura de Metodología de la Investigación, se conseguirá construir un aprendizaje significativo que permitirá generar cambios de actitudes para una gestión adecuada del riesgo en sus contextos habituales".

\section{Resultados del plan de acción}

Para la realización del plan de la unidad didáctica, primero se hizo una revisión cuidadosa del programa de la Asignatura de Metodología de la Investigación, encontrándose que en ninguna unidad temática de las tres unidades de éste, se ha integrado la gestión de riesgo y cambio climático, por tanto, para lograr insertar esta temática como eje transversal, se hizo necesario hacer ajustes en los objetivos de la unidad y de los contenidos a tomar en cuenta para dicha inserción, así como la planificación didáctica de los mismos.

La unidad didáctica se dividió en diez sesiones de trabajo diseñadas de esta manera para poder desarrollar cada uno de los contenidos y actividades correspondientes a la unidad seleccionada, llevándose a cabo en un periodo de tres meses: abril - junio del año 2013.

Las actividades y/o estrategias de aprendizaje realizadas fueron: 
- Presentación de las líneas de investigación.

- Clase expositiva-interactiva por parte del facilitador.

- Trabajo cooperativo: Grupos de investigación, clase expositiva por parte de los/as estudiantes, clases prácticas.

- Entrega y defensa del trabajo final.

El objetivo general del plan de acción era lograr que los y las estudiantes apliquen el proceso de Investigación Científica en problemas relacionados a la gestión de riesgo y cambio climático con enfoque de género y gobernabilidad, desde la Asignatura Metodología de la Investigación, a través de los siguientes objetivos específicos:

- Identificar la línea de investigación que les corresponde desarrollar de acuerdo al perfil de su carrera.

- Ejecutar las etapas y pasos del proceso de investigación integrando la gestión de riesgo y cambio climático con enfoque de género y gobernabilidad.

- Evaluar el trabajo realizado por los equipos de investigación.

En base a la acción realizada se considera pertinente decir, que las diferentes actividades desarrolladas con los grupos de estudiantes, resultaron de gran provecho para enriquecer el proceso de investigación y valorar la importancia de la misma para la solución de problemas socioeducativos. Asimismo, se destacan aspectos positivos alrededor de la transversalización del eje de gestión de riesgo y cambio climático con enfoque de género y gobernabilidad, en las diferentes temáticas de investigación abordadas por los grupos de estudiantes. Los resultados obtenidos con las acciones realizadas, reflejan los siguientes logros:

Se logró integrar el eje transversal de gestión de riesgo y cambio climático en la mayoría de las temáticas de investigación elegidas por los grupos de estudiantes (de 14 trabajos de investigación propuestos, 9 aplicaron el proceso investigativo a problemáticas relacionadas con la gestión de riesgo, integrando también algunos de ellos, el cambio climático y los enfoques de género y gobernabilidad).

"La integración se realizó desde el momento que se identificó el problema, logrando adecuar la gestión de riesgo en el tema, nos apropiamos de herramientas necesarias para realizar un trabajo investigativo".

Dos equipos de investigación enfocaron de manera directa su proyecto de Investigación en la línea de investigación Calidad Educativa, específicamente en el tema: Elaboración e implementación de planes de mitigación, prevención de riesgo ante desastres en centros de Educación Preescolar, Primaria y Secundaria.

"Este trabajo nos permitirá hacer una incidencia en el cambio de actitudes de los docentes para implementarlo en el instituto que enfrenta serios problemas de riesgos ante desastres"...

Otro logro significativo fue que los/as estudiantes de Ciencias Naturales reconocieran que aun cuando estudian una carrera directamente relacionada con las temáticas de gestión de riesgo y cambio climático, éstas no se les había incluido en las diferentes asignaturas. Situación que evidencia actitudes positivas en torno a la problemática, considerándola importante y necesaria para su formación.

"Un logro fue enriquecer nuestros conocimientos acerca de gestión de riesgo ya que poseemos poca información"...

El amplio conocimiento que tenían los/as estudiantes de tercer año de Ciencias Naturales sobre la temática a transversalizar, contribuyó de manera positiva durante el proceso. 
Producto de la calidad del trabajo realizado por la mayoría de los equipos de investigación, seis de los trabajos presentados fueron seleccionados para presentarse en la Jornada Universitaria de Desarrollo Científico 2013 (JUDC), despertando bastante motivación en los/as estudiantes.

\section{CONCLUSIONES Y COMPROMISOS}

Como resultado del trabajo de investigación-Acción realizado se logró comprobar lo planteado por Herrera (2013) acerca de que los ejes transversales son realidades educativas que deben impregnar dinámicamente el currículo, siendo el propósito de estos, formar personas con autonomía moral e intelectual, capaces de comprometerse consigo y con las demás.

En este sentido, y en correspondencia con el propósito formulado, se concluye que la temática de gestión de riesgo y cambio climático con enfoque de género y gobernabilidad, se puede integrar como eje transversal en la unidad dos Proceso de la Investigación Científica, de la Asignatura Metodología de la Investigación, de la carrera de Enseñanza de las Ciencias Naturales, planificando adecuadamente la aplicación del proceso de la Investigación (etapas y pasos) en problemas relacionados a dicha temática. Logrando con ello, construir aprendizajes significativos en los/as estudiantes, que les permitirá generar una cultura de gestión de riesgo en sus contextos habituales.

Por tanto, se puede decir que se comprobó la hipótesis de acción.

\section{Compromisos para mejorar la acción}

Para seguir avanzando en este proceso y en base a las dificultades surgidas durante el mismo, se considera necesario:

- Garantizar que los/as estudiantes le den seguimiento a sus proyectos de investigación en la Asignatura de Investigación Aplicada.

- Seguir implementando la experiencia de inserción del eje en la Asignatura Metodología de la Investigación, pero esta vez procurar que todos/ as los/as estudiantes integren la temática con sus diferentes enfoques en sus diferentes trabajos de investigación.

- Planificar la incorporación del eje desde el inicio del curso.

- Compartir esta experiencia metodológica con los diferentes docentes de Metodología de la Investigación, a fin de promover la integración de la temática de gestión de riesgo y cambio climático con enfoque de género y gobernabilidad, en la Asignatura Metodología de la Investigación de las diferentes carreras del Departamento de Ciencias de la Educación y Humanidades.

\section{BIBLIOGRAFÍA}

Blandón, D. Y. (2013). Modulo dos Introduccion a la Gestión de Riesgo. Diplomado Gestión de Riesgo y Cambio Climático. Centro de Formación y Capacitación Permanente para la Gestión Integral de Riesgos y Desastres. UNAN- Managua/ FAREM-Estelí.

Herrera, E. (2013). Modulo doce Curso de Investigación Acción. Diplomado Gestión de Riesgo y Cambio Climático. Centro de Formación y Capacitación Permanente para la Gestión Integral de Riesgos y Desastres. UNAN- Managua/FAREM-Estelí.

Herrera, E. (2013). Aplicación del eje transversal de gestión de riesgo www.slideshare.net. Recuperado el 20 de junio de 2013, de http://www.slideshare. net/nanli1612/ejes-transversales-16385303

Milán. J. A. (2009). Apuntes sobre el cambio climático en Nicaragua (primera ed.). Managua, Nicaragua. Sampieri, R. H. (2013). Metodologia de la Investigación. México, México: Mc Graw Hill. maes Facil Manual de Investigación (Vol. I). Managua: Universitaria. 